Jolanta Wesołowska, Instytut Matematyki, Uniwersytet Gdaǹski, ul. Wita

Stwosza 57, 80-952 Gdaǹsk, Poland. e-mail: jwes@ksinet.univ.gda.pl

\title{
ON SETS OF CONVERGENCE POINTS OF SEQUENCES OF SOME REAL FUNCTIONS
}

\begin{abstract}
The purpose of this paper is to study a set of convergence points of sequence of real functions from a given class. Here, continuous functions, Borel measurable functions, approximately continuous functions and derivatives are considered.
\end{abstract}

The investigation of some sets determined by sequences of functions is motivated by the well-known result due to Hahn [1] and also Sierpiński [7] stating that a subset $A$ of a Polish space $X$ is of type $\mathcal{F}_{\sigma \delta}$ iff there exists a sequence $\left\{f_{n}: n \in \mathbb{N}\right\} \subset \mathbb{R}^{X}$ of continuous functions such that $A=\{x \in$ $X:\left(f_{n}(x)\right)_{n}$ converges $\}$ (see also [2, Theorem 23.18, p. 185]). It seems interesting to find the analogous characterization of sets of convergence points for sequences of functions from other classes. The same problem and its connections with additional set-theoretic axioms has been considered for transfinite sequences of functions in [4].

In the present paper the sequences of functions of Baire class $\alpha$, approximately continuous functions and derivatives are examined. All functions considered here are real functions defined on the real line $\mathbb{R}$. Throughout this paper the following abbreviations for some classes of subsets of $\mathbb{R}$ will be used:

$\Pi_{1}^{0}\left(\Sigma_{1}^{0}\right)$ — closed (open) subsets of $\mathbb{R} ;$

$\Pi_{\alpha}^{0}\left(\Sigma_{\alpha}^{0}\right)$ - the multiplicative (additive) class $\alpha$ of Borel sets, $0<\alpha<\omega_{1}$;

$\mathcal{M}$ - the $\sigma$-ideal of meager (first-category) subsets of $\mathbb{R}$;

$\mathcal{T}_{d}$ — the density topology (recall that $\mathcal{T}_{d}$ consists of all Lebesgue measurable sets having density 1 at each of its points [5]).

Key Words: sequence of functions, sets of convergence points, Baire class $\alpha$ functions, approximately continuous functions.

Mathematical Reviews subject classification: Primary: 26A21; Secondary: 26A03.

Received by the editors October 28, 1999

* Supported by University of Gdańsk, grant BW Nr 5100-5-0258-9. 
Recall some definitions of various types of functions $f: \mathbb{R} \rightarrow \mathbb{R}$ which are investigated. Each class of functions is denoted by symbol on the left.

$\mathcal{B}_{\alpha}-f$ is of Baire class $\alpha$, where $\alpha<\omega_{1}$ iff for every open set $U \subset \mathbb{R}$ $f^{-1}(U) \in \Sigma_{\alpha+1}^{0}$;

$l_{1}\left(u_{1}\right)-f$ is lower (resp. upper) semicontinuous iff it is a pointwise limit of non-decreasing (resp. non-increasing) sequence of continuous functions;

$l_{\alpha}\left(u_{\alpha}\right)-f$ is of Young lower (resp. upper) class $\alpha$, where $0<\alpha<\omega_{1}$ iff it is a pointwise limit of non-decreasing (resp. non-increasing) sequence $\left\{f_{n}: n \in \mathbb{N}\right\} \subset \bigcup_{\beta<\alpha} u_{\beta}\left(\operatorname{resp} . \bigcup_{\beta<\alpha} l_{\beta}\right)$;

$\mathcal{A}-f$ is approximately continuous iff for every open set $U \subset \mathbb{R} f^{-1}(U) \in \mathcal{T}_{d}$.

Moreover, we denote by $b \mathcal{A}$ the class of all bounded approximately continuous functions and by $\Delta$ the class of all derivatives.

Denote by $L\left(\left\{f_{n}: n \in \mathbb{N}\right\}\right)$ a set of all convergence points of a sequence $\left\{f_{n}: n \in \mathbb{N}\right\} \subset \mathbb{R}^{\mathbb{R}}$, i.e.

$$
L\left(\left\{f_{n}: n \in \mathbb{N}\right\}\right)=\left\{x \in \mathbb{R}:\left\{f_{n}(x): n \in \mathbb{N}\right\} \text { converges }\right\} .
$$

\section{Remark 1.}

$$
L\left(\left\{f_{n}: n \in \mathbb{N}\right\}\right)=\bigcap_{m \in \mathbb{N}} \bigcup_{n \in \mathbb{N}} \bigcap_{k \in \mathbb{N}}\left\{x \in \mathbb{R}:\left|f_{n+k}(x)-f_{n}(x)\right| \leq 1 / m\right\} .
$$

For a family of functions $\mathcal{F} \subset \mathbb{R}^{\mathbb{R}}$ define

$$
\mathcal{L}(\mathcal{F})=\left\{L\left(\left\{f_{n}: n \in \mathbb{N}\right\}\right):\left\{f_{n}: n \in \mathbb{N}\right\} \subset \mathcal{F}\right\} .
$$

In this language the theorem obtained by Hahn and Sierpiński takes the following form.

Theorem 1. For the family $\mathcal{C}$ of continuous functions $\mathcal{L}(\mathcal{C})=\Pi_{3}^{0}$.

To obtain its generalization onto the case of functions of Baire class $\alpha$ we will use some well-known facts.

Theorem 2. [8, Theorem 19, p. 30]

Let $\alpha<\omega_{1}$ and $f: \mathbb{R} \rightarrow[-\infty,+\infty]$. Then

- $f \in l_{\alpha}$ iff $f^{-1}((a, \infty)) \in \Sigma_{\alpha}^{0}$ for each $a \in \mathbb{R}$;

- $f \in u_{\alpha}$ iff $f^{-1}((-\infty, a)) \in \Sigma_{\alpha}^{0}$ for each $a \in \mathbb{R}$. 
Remark 2. For $\alpha<\omega_{1}, \mathcal{B}_{\alpha}=l_{\alpha+1} \cap u_{\alpha+1}$.

Theorem 3. For $\alpha<\omega_{1}, \mathcal{L}\left(\mathcal{B}_{\alpha}\right)=\Pi_{\alpha+3}^{0}$.

Proof. Fix $\alpha<\omega_{1}$. The inclusion " $\subset$ " follows from Remark 1 and the definition of $\mathcal{B}_{\alpha}$ functions. So it remains to show that if $A \in \Pi_{\alpha+3}^{0}$, then $A=L\left(\left\{f_{n}: n \in \mathbb{N}\right\}\right)$ for some sequence $\left\{f_{n}: n \in \mathbb{N}\right\} \subset \mathcal{B}_{\alpha}$. This follows by the same method as in $\left[2\right.$, Theorem 23.18, p. 185]. Since $A \in \Pi_{\alpha+3}^{0}$, we have $A=\bigcap_{m \in \mathbb{N}} A_{m}$, where $A_{m} \in \Sigma_{\alpha+2}^{0}$. First, suppose that

(1) for every $m \in \mathbb{N}$ and $A_{m} \in \Sigma_{\alpha+2}^{0}$ there is a sequence $\left\{f_{n}: n \in \mathbb{N}\right\} \subset \mathcal{B}_{\alpha}$ such that

- $\left|f_{n}^{m}(x)\right| \leq 1 / m$ for $n \in \mathbb{N}$ and $x \in \mathbb{R}$,

- $A_{m}=L\left(\left\{f_{n}^{m}: n \in \mathbb{N}\right\}\right)=\left\{x \in \mathbb{R}: \lim _{n} f_{n}^{m}(x)=0\right\}$.

Then rewrite $\bigcup_{m \in \mathbb{N}}\left\{f_{n}^{m}: n \in \mathbb{N}\right\}$ as a single sequence $\left\{f_{i}: i \in \mathbb{N}\right\}$. Of course, $\lim _{i} f_{i}(x)=0$ for all $x \in A$. To see this fix $x \in A, \varepsilon>0$ and take $k \in \mathbb{N}$ such that $1 / k<\varepsilon$. Then there is a positive integer $i_{0}=i_{0}(\varepsilon)$ such that $\left|f_{n}^{m}(x)\right|<\varepsilon$ for $n>i_{0}$ and $m \leq k$. But for $m>k\left|f_{n}^{m}(x)\right| \leq 1 / m<1 / k<\varepsilon$, so $\left|f_{i}(x)\right|<\varepsilon$ for $i>i_{0}$. On the other hand, if $x \notin A$, then $x \notin A_{m_{0}}$ for some $m_{0} \in \mathbb{N}$ and consequently $\left\{f_{n}^{m_{0}}(x): n \in \mathbb{N}\right\}$ diverges, so $\left\{f_{i}(x): i \in \mathbb{N}\right\}$ diverges too.

What is left is to show (1). Fix $m \in \mathbb{N}$ and $A_{m} \in \Sigma_{\alpha+2}^{0}$. Then $A_{m}=$ $\bigcup_{n \in \mathbb{N}} F_{n}^{m}$, where $F_{n}^{m} \in \Pi_{\alpha+1}^{0}$ and $F_{n}^{m} \subseteq F_{n+1}^{m}$ for $n \in \mathbb{N}$. Consider the following real function $g: \mathbb{R} \rightarrow[1,+\infty]$ :

$$
g(x)= \begin{cases}1 & \text { if } x \in F_{1}^{m} \\ n & \text { if } x \in F_{n}^{m} \backslash F_{n-1}^{m} \text { for } n \geq 2 \\ +\infty & \text { if } x \in \mathbb{R} \backslash A_{m} .\end{cases}
$$

For $a \in \mathbb{R}$ we have $\{x \in \mathbb{R}: g(x)>a\}=\mathbb{R}$ if $a<1$ or $\{x \in \mathbb{R}: g(x)>a\}=$ $\mathbb{R} \backslash F_{n}^{m} \in \Sigma_{\alpha+1}^{0}$ if $n \leq a<n+1$ for $n \geq 1$ and consequently $g \in l_{\alpha+1}$, by Theorem 2. It follows that there is a non-decreasing sequence $\left\{g_{n}: n \in \mathbb{N}\right\} \subset$ $\mathcal{B}_{\alpha}$ pointwise convergent to $g$. For each $n \in \mathbb{N}$ put $\varphi_{n}=\min \left\{n, \max \left\{g_{n}, 1\right\}\right\}$. Then $1 \leq \varphi_{n} \leq n$ and $\varphi_{n+1}-\varphi_{n} \leq n$. Clearly, $\varphi_{n} \in \mathcal{B}_{\alpha}$ and $\lim _{n} \varphi_{n}=$ $g$. Moreover, we can interpolate between $\varphi_{n}$ and $\varphi_{n+1}$ the functions $p_{k}^{n}=$ $\varphi_{n}+\frac{k}{2 n}\left(\varphi_{n+1}-\varphi_{n}\right)$ for $k=0,1, \ldots, 2 n$. By renumbering we have a sequence $\left\{p_{n}: n \in \mathbb{N}\right\} \subset \mathcal{B}_{\alpha}$ such that $1=p_{0} \leq p_{1} \leq p_{2} \leq \ldots, p_{n+1}-p_{n} \leq 1 / 2$ and $f=\lim _{n} p_{n}$. Putting $f_{n}^{m}=\frac{1}{m} \sin \left(\pi p_{n}\right)$ we obtain the sequence satisfying (1).

The next result deals with the case of approximately continuous functions. 
Lemma 1. (cf. [3, Lemma 5]) Every set $A \in \Pi_{2}^{0}$ is a countable union of $\Pi_{2}^{0}$ sets closed in the density topology.

Proof. There are closed (in the usual sense) sets $F_{n}, n \in \mathbb{N}$ such that $F=$ $\bigcup_{n \in \mathbb{N}} F_{n} \subset A$ and $A \backslash F$ has Lebesgue measure zero. Put $A_{n}=F_{n} \cup(A \backslash F)$. Then every $A_{n}$ is closed in $\mathcal{T}_{d}$ and $A=\bigcup_{n \in \mathbb{N}} A_{n}$. Moreover, $A_{n}=F_{n} \cup(A \cap$ $(\mathbb{R} \backslash F)) \in \Pi_{2}^{0}$.

Corollary 1. Every set $A \in \Pi_{4}^{0}$ can be represented in the form $A=\bigcap_{m \in \mathbb{N}} A_{m}$, where for $m \in \mathbb{N} A_{m}$ is a countable union of $\Pi_{2}^{0}$ sets closed in the density topology.

Lemma 2. [9, Lemma 11, p. 26] For every set $A \in \mathcal{T}_{d} \cap \Sigma_{2}^{0}$ there is an approximately continuous function $f: \mathbb{R} \rightarrow \mathbb{R}$ such that $f(x) \in(0,1]$ if $x \in A$ and $f(x)=0$ if $x \notin A$.

Lemma 3. [6] If $g: \mathbb{R} \rightarrow \mathbb{R}$ is a positive function such that for every $a \in \mathbb{R}$ $\{x \in \mathbb{R}: g(x)>a\} \in \mathcal{T}_{d} \cap \Sigma_{2}^{0}$, then $g$ is a pointwise limit of a non-decreasing sequence of approximately continuous functions. ${ }^{1}$

Proof. Enumerate the set of all positive rational numbers as $\left\{q_{k}: k \in \mathbb{N}\right\}$. Fix $k \in \mathbb{N}$ and define $E_{k}=\left\{x \in \mathbb{R}: g(x)>q_{k}\right\}$. Then $E_{k} \in \mathcal{T}_{d} \cap \Sigma_{2}^{0}$ and by Lemma 2 there is an approximately continuous function $p_{k}: \mathbb{R} \rightarrow \mathbb{R}$ such that $0<p_{k} \leq 1$ on $E_{k}$ and $p_{k}=0$ on $\mathbb{R} \backslash E_{k}$. For $n \in \mathbb{N}$ put $p_{n}^{k}=\min \left\{q_{k}, n p_{k}\right\}$. Then $q_{k} \cdot \chi_{E_{k}}$ is a pointwise limit of non-decreasing sequence $\left\{p_{n}^{k}: n \in \mathbb{N}\right\} \subset \mathcal{A}$ and $p_{n}^{k}<g$. Let $g_{n}=\max _{1 \leq k \leq n} p_{n}^{k}$. It is easy to check that:

(i) $g_{n} \in \mathcal{A}$,

(ii) $g_{n} \leq g_{n+1}<g$,

(iii) if $g(x)>q$ for some positive rational $q$, then $\lim _{n} g_{n} \geq q$.

It follows that $\lim _{n} g_{n}=g$ and $\left\{g_{n}: n \in \mathbb{N}\right\}$ is a required sequence.

Remark 3. Lemma 3 holds also for $g: \mathbb{R} \rightarrow[0,+\infty]$

Now, we are able to prove the following.

Theorem 4. $\mathcal{L}(\mathcal{A})=\Pi_{4}^{0}$

\footnotetext{
${ }^{1}$ This fact was announced during the conference Summer School on Real Functions Theory in Liptovský Ján (Slovakia) in 1998.
} 
Proof. It is well-known that $\mathcal{A} \subset \mathcal{B}_{1}$ (see, e. g. [3]), so the inclusion " $\subset$ " follows from Theorem 3. Now, fix $A \in \Pi_{4}^{0}$. We claim that $A=L\left(\left\{f_{n}: n \in \mathbb{N}\right\}\right)$ for some sequence $\left\{f_{n}: n \in \mathbb{N}\right\}$ of approximately continuous functions. To show this we apply the method similar to that in the proof of Theorem 3. By Corollary $1 A=\bigcap_{m \in \mathbb{N}} A_{m}$, where for $m \in \mathbb{N} A_{m}=\bigcup_{n \in \mathbb{N}} F_{n}^{m}, F_{n}^{m} \in \Pi_{2}^{0}$, $F_{1}^{m} \subseteq F_{2}^{m} \subseteq \ldots$ and $\mathbb{R} \backslash F_{n}^{m} \in \mathcal{T}_{d}$. Fix $m \in \mathbb{N}$. Then $g: \mathbb{R} \rightarrow[1,+\infty]$ given by the formula

$$
g(x)= \begin{cases}1 & \text { if } x \in F_{1}^{m} \\ n & \text { if } x \in F_{n}^{m} \backslash F_{n-1}^{m} \text { for } n \geq 2 \\ +\infty & \text { if } x \in \mathbb{R} \backslash A_{m}\end{cases}
$$

satisfies the assumptions of Lemma 3. Consequently, there is a non-decreasing sequence $\left\{g_{n}: n \in \mathbb{N}\right\} \subset \mathcal{A}$ pointwise convergent to $g$. The same construction as before gives us a sequence $\left\{f_{n}^{m}: n \in \mathbb{N}\right\} \subset \mathcal{A}$ such that $\left|f_{n}^{m}(x)\right| \leq 1 / m$ for $n \in \mathbb{N}, x \in \mathbb{R}$ and $A_{m}=L\left(\left\{f_{n}^{m}: n \in \mathbb{N}\right\}\right)=\left\{x \in \mathbb{R}: \lim _{n} f_{n}^{m}(x)=0\right\}$. Finally, it is enough to rewrite $\bigcup_{m \in \mathbb{N}}\left\{f_{n}^{m}: n \in \mathbb{N}\right\}$ as a single sequence $\left\{f_{i}: i \in\right.$ $\mathbb{N}$ \} to obtain the one we claimed.

The last result is a consequence of Theorems 3 and 4 .

Theorem 5. $\mathcal{L}(\Delta)=\Pi_{4}^{0}$

Proof. Note that the construction in Theorem 4 uses bounded approximately continuous functions, so we have actually proved that $\mathcal{L}(b \mathcal{A})=\Pi_{4}^{0}$. Since $b \mathcal{A} \subset \Delta \subset \mathcal{B}_{1}$ (see e. g. [3]), the proof is complete.

Acknowledgments. The author wishes to express her thanks to the referee for pointing out the consequences of Theorem 4 for derivatives.

\section{References}

[1] H. Hahn, Über die Menge der Konvergenzpunkte einer Funktionfolge, Archiv. der Math. und Physik 28(1919), 34-45.

[2] A. S. Kechris, Classical Descriptive Set Theory, Springer, New York 1995.

[3] M. Laczkovich, G. Petruska, A theorem on approximately continuous functions, Acta Math. Acad. Sci. Hungar. 24(1973), 383-387.

[4] T. Natkaniec, J. Wesolowska, On the convergence of $\omega_{1}$ sequences of real functions, to appear in Acta Math. Hungar. 91(4)(2001).

[5] J. C. Oxtoby, Measure and Category, Springer-Verlag, New York, 1971. 
[6] F. Prus-Wiśniowski, G. Szkibiel, A note on SAC property, to appear in Tatra Mount. Math. Public.

[7] W. Sierpiński, Sur l'ensemble des points de convergence d'une suite de fonctions continues, Fund. Math. 2(1921), 41-49.

[8] W. Sierpiński, Funkcje przedstawialne analitycznie, Warszawa, 1925.

[9] Z. Zahorski, Sur la première dérivée, Trans. Amer. Math. Soc. 69(1950), 1-54. 